\title{
Erratum to: DNA methylome profiling of human tissues identifies global and tissue-specific methylation patterns
}

Kaie Lokk ${ }^{1,2,3}$, Vijayachitra Modhukur ${ }^{4}$, Balaji Rajashekar ${ }^{4}$, Kaspar Märtens $^{4}$, Reedik Mägi ${ }^{5}$, Raivo Kolde ${ }^{4}$, Marina Koltšina ${ }^{1}$, Torbjörn K. Nilsson ${ }^{6}$, Jaak Vilo ${ }^{4}$, Andres Salumets ${ }^{7,8,9^{*}}$ and Neeme Tönisson ${ }^{1,2,5^{*}}$

\section{Erratum}

After the publication of this work [1] it was noticed that Additional file 1 is the same as Additional file 2.

Additional file 1 has now been correctly replaced.

\section{Additional file}

Additional file 1: Methylation calidation using Sanger sequencing. For validation of the methylation data from BeadChip, 17 genes were chosen, including unmethylated sites $(n=1)$, fully methylated sites $(n=2)$, and genes with tDMRs $(n=14)$ representing $36 \mathrm{CpG}$ sites altogether. The $x$-axis shows DNA methylation beta-values obtained from BeadChip, and the $y$-axis shows beta values from Sanger sequencing. (PDF 24 kb)

\section{Author details}

${ }^{1}$ Institute of Molecular and Cell Biology, University of Tartu, Tartu, Estonia. ${ }^{2}$ Department of Genetics, United Laboratories, Tartu University Hospital, Tartu, Estonia. ${ }^{3}$ Estonian Biocentre, Tartu, Estonia. ${ }^{4}$ Institute of Computer Science, University of Tartu, Tartu, Estonia. ${ }^{5}$ Estonian Genome Center, University of Tartu, Tartu, Estonia. ${ }^{6}$ Department of Medical Biosciences, Clinical Chemistry, Umeå University, Umeå, Sweden. ${ }^{7}$ Competence Centre on Reproductive Medicine and Biology, Tartu, Estonia. ${ }^{8}$ Department of Obstetrics and Gynecology, University of Tartu, Tartu, Estonia. ${ }^{9}$ Institute of Bio- and Translational Medicine, University of Tartu, Tartu, Estonia.

Received: 25 October 2016 Accepted: 25 October 2016 Published online: 01 November 2016

\section{Reference}

1. Lokk K, Modhukur V, Rajashekar B, Märtens K, Mägi R, Kolde R, et al. DNA methylome profiling of human tissues identifies global and tissue-specific methylation patterns. Genome Biol. 2014;15:r54.

\footnotetext{
*Correspondence: andres.salumets@ccrmb.ee; neemet@ut.ee

${ }^{7}$ Competence Centre on Reproductive Medicine and Biology, Tartu, Estonia ${ }^{1}$ Institute of Molecular and Cell Biology, University of Tartu, Tartu, Estonia
} 